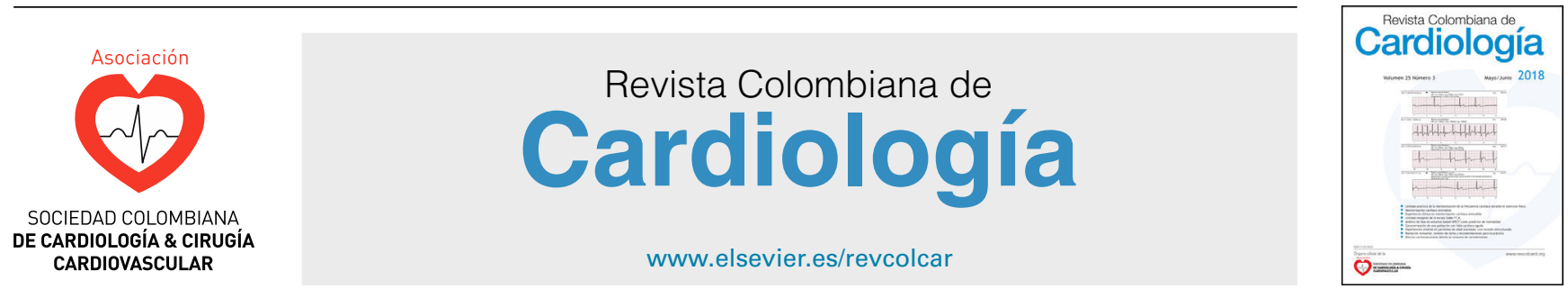

IMÁGENES EN CARDIOLOGÍA

\title{
Utilidad de técnicas de imagen en la valoración de la enfermedad coronaria - Tomografía de coherencia óptica
}

\author{
Natalia Pinilla Echeverri*, Matthew Sibbald y Tej Sheth
}

McMaster University, Hamilton Health Sciences, Hamilton, Canada

Disponible en Internet el 3 de julio de 2019

\section{PALABRAS CLAVE \\ Tomografía de coherencia óptica; \\ Aterosclerosis \\ coronaria; \\ Lesión culpable; \\ Placa vulnerable}

\begin{abstract}
Resumen La tomografía de coherencia óptica es una técnica de imagen intracoronaria de alta resolución, que permite la valoración intraluminal y transmural de las estructuras del vaso coronario. Ofrece una penetración de tejido en escala milimétrica $(1-2 \mathrm{~mm})$ y una resolución espacial longitudinal y lateral en escala micrométrica. La resolución está en el orden de 10$20 \mu \mathrm{m}$, aproximadamente diez veces superior a la resolución del ultrasonido intravascular que está en el orden de 100-150 $\mu \mathrm{m}$ y que es otra técnica de imagen intracoronaria con amplio uso en la práctica clínica actual.

Desde su introducción hace más de dos décadas ha tenido un uso incremental tanto en investigación como en la práctica clínica. Ha sido especialmente útil en la evaluación de la historia natural de la aterosclerosis, la descripción de los mecanismos de síndrome coronario agudo y la optimización del intervencionismo coronario. Hoy en día tiene especial utilidad en el diagnóstico de la lesión culpable en casos en los que la angiografía es limitada, en la planificación de intervencionismo percutáneo complejo (enfermedad difusa, bifurcaciones, lesiones calcificadas) y en casos de fallo del stent (trombosis y restenosis); que es quizás una de las indicaciones por excelencia, con el objetivo de entender la fisiopatología y proveer el tratamiento percutáneo adecuado.

En esta revisión se hará énfasis en las consideraciones para usar tomografía de coherencia óptica como técnica de imagen intracoronaria en el diagnóstico y clasificación de enfermedad aterosclerótica.

Crown Copyright (c) 2019 Publicado por Elsevier España, S.L.U. en nombre de Sociedad Colombiana de Cardiología y Cirugía Cardiovascular. Este es un artículo Open Access bajo la licencia CC BY-NC-ND (http://creativecommons.org/licenses/by-nc-nd/4.0/).
\end{abstract}

\footnotetext{
* Autor para correspondencia.

Correo electrónico: pinillan@mcmaster.ca (N. Pinilla Echeverri).
} 


\section{KEYWORDS}

Optic coherence tomography; Coronary atherosclerosis; Culprit lesion; Vulnerable plaque

\section{Usefulness of imaging techniques in the assessment of coronary disease - Optic Coherence Tomography}

\begin{abstract}
Optic coherence tomography is a high resolution imaging technique that enables the lumen and the walls of the structures of the coronary vessel to be examined. It offers a tissue penetration on a millimetre scale $(1-2 \mathrm{~mm})$ and a longitudinal and lateral spatial resolution on a micrometre scale. The resolution is in the order of $10-20 \mu \mathrm{m}$, approximately 10 times superior to the resolution of intravascular ultrasound, which is in the order of $100-150 \mu \mathrm{m}$, and is another intra-coronary imaging technique widely used in current clinical practice.

Since its introduction more than 20 years ago, it has been increasingly used in research, as well as in clinical practice. It has been especially useful in the evaluation of the natural history of atherosclerosis, the description of the mechanisms of acute coronary syndrome, and the optimisation of coronary interventions. It currently has a special use in the diagnosis of culprit lesions in cases where angiograph is limited, in the planning of complex percutaneous catheterisation (diffuse diseases, bifurcations, and calcified lesions), and in cases of stent failures (thrombosis and re-stenosis). The latter is probably one of the par excellence indications, with the aim of understanding the pathophysiology and to provide the appropriate percutaneous treatment.

In this review, emphasis will be made on the considerations for using optic coherence tomography as an intracoronary imaging technique in the diagnosis and classification of atherosclerotic disease.

Crown Copyright $\odot 2019$ Published by Elsevier España, S.L.U. on behalf of Sociedad Colombiana de Cardiología y Cirugía Cardiovascular. This is an open access article under the CC BY-NC-ND license (http://creativecommons.org/licenses/by-nc-nd/4.0/).
\end{abstract}

\section{Introducción}

La angiografía es una técnica de imagen bidimensional que provee un luminograma de la arteria coronaria y que sigue siendo la técnica más usada para el diagnóstico de enfermedad coronaria y para guiar el intervencionismo percutáneo; sin embargo, posee reconocidas limitaciones que nacen en el concepto de valoración de una estructura tridimensional, como es la arteria coronaria, con una imagen bidimensional que requiere múltiples imágenes con diferentes angulaciones ${ }^{1}$. La angiografía está asociada a alta variabilidad inter-observador en la estimación visual de la estenosis coronaria, incluso entre operadores experimentados ${ }^{2}$, y el análisis cuantitativo por quantitative coronary analysis (QCA) solo mejora marginalmente la aproximación diagnóstica funcional ${ }^{3}$. Como es de esperarse en una imagen bidimensional, no ofrece ninguna información de la caracterización de la placa subyacente ni estimación de la distribución de la enfermedad coronaria, ya que inicialmente la acumulación de la placa expande la lámina elástica externa como mecanismo compensatorio para preservar el área luminal (fenómeno de remodelado positivo) lo cual puede aparentar en angiografía una arteria sin alteraciones en su diámetro cuando sus paredes presentan, en efecto, diferentes estadios de aterosclerosis ${ }^{4}$.

La imagen intracoronaria por ultrasonido y por tomografía de coherencia óptica, provee una visualización minuciosa de la arteria coronaria y supera así muchas de las limitaciones de la angiografía. Comparado con la imagen en escala de grises del utrasonido intravascular, la imagen de tomografía de coherencia óptica es de adquisición más rápida, mayor resolución y menor profundidad de penetración en el tejido.
La alta resolución espacial de la tomografía de coherencia óptica ofrece una imagen detallada que permite la caracterización de tejidos de la pared e intracoronarios (lípidos, tejido fibrótico, calcio, neoíntima, trombo); además, ofrece una valoración precisa de la implantación del stent como aposición, expansión, prolapso de tejido y disección de los bordes $^{5}$.

La tomografía de coherencia óptica es una modalidad de imagen que es análoga a la imagen de ultrasonido, pero utiliza luz en lugar de sonido. Las imágenes de sección transversal se generan midiendo el eco, el retraso de tiempo y la intensidad de la luz reflejada o dispersada por las estructuras internas del tejido. Mejora la localización del origen de la señal devuelta debido a la longitud de onda mucho más corta de la luz cuando se compara con el ultrasonido; por tanto, ofrece una resolución significativamente superior. Como la velocidad de la luz no permite la medición directa del retardo del eco, se han utilizado técnicas interferométricas para analizar la señal luminosa reflejada ${ }^{6}$.

El catéter de tomografía de coherencia óptica se puede acercar en diseño y tamaño a un microcatéter con la excepción de que tiene un lente de fibra óptica encapsulado en un cable metálico de torsión que está diseñado para girar a altas velocidades y permitir adecuada adquisición de imágenes transversales. El catéter es compatible con una guía de angioplastia convencional de $0,014^{\prime \prime}$, que se inserta en un corto monorriel en la punta del catéter. El lente óptico se debe localizar distal al segmento coronario de interés y una vez activada la adquisición de imagen, el catéter gira a una velocidad rápida que permite capturar hasta 180 imágenes por segundo con una resolución axial por debajo de $20 \mu \mathrm{m}$. Existen dos posibles longitudes de adquisición: $54 \mathrm{~mm}$ o de 
alta resolución, que permite obtener 540 imágenes de sección transversal consecutivas; y $75 \mathrm{~mm}$ o de reconocimiento, que permite obtener 375 imágenes transversales ${ }^{7}$.

La ejecución de imagen intracoronaria con tomografía de coherencia óptica requiere el mismo equipo que para el intervencionismo coronario y no supone un riesgo adicional para el procedimiento ni para el paciente más allá de lo que se requiere para el propio intervencionismo. El evento adverso más frecuente es el espasmo coronario transitorio, que se resuelve fácilmente mediante la administración de nitroglicerina. La tomografía de coherencia óptica requiere inyección de contraste yodado para la adquisición de las imágenes ya que la luz no puede penetrar los glóbulos rojos, por consiguiente no se considerará en pacientes con enfermedad renal aguda o crónica que puedan empeorar con la administración de contraste ${ }^{7}$.

Desde los inicios de la tecnología hace más de dos décadas, la tomografía de coherencia óptica se ha empleado ampliamente en investigación clínica para describir in vivo todos esos fenómenos que ya eran bien reconocidos por la patología en cuanto al proceso de aterosclerosis, los mecanismos de síndrome coronario agudo y la aterosclerosis intrastent; sin embargo, hoy día tiene amplia utilidad en la práctica clínica.

Este documento tiene como objetivo describir el potencial de tomografía de coherencia óptica en la cualificación y cuantificación de las placas coronarias y el reconocimiento del proceso de aterosclerosis.

\section{Clasificación y definición de las lesiones ateroscleróticas por tomografía de coherencia óptica}

La tomografía de coherencia óptica es la técnica de imagen intracoronaria más útil para describir la morfología de las placas coronarias y la clasificación de placa vulnerable ya que su alta resolución permite la medición de la capa fibrosa. La caracterización del tejido aterosclerótico por coherencia óptica es posible gracias a la valoración de retrodispersión y atenuación de los tejidos. Para reconocer con claridad las diferentes placas y estadios de la aterosclerosis, es necesario familiarizarse con la imagen de la arteria coronaria normal, una estructura trilaminar conformada por capa íntima, media y adventicia separadas por las láminas elásticas interna y externa (fig. 1A). A partir de allí, según la intensidad y la atenuación de la luz, es posible definir las tres placas coronarias principales:

- Lipídica: placa con señal de alta intensidad (brillante), alta atenuación (la señal de la luz se pierde rápidamente al atravesar la placa), con transición difusa entra la capa íntima y la placa lipídica (fig. 1B).

- Fibrosa: placa homogénea con señal de alta intensidad y baja atenuación (fig. 1C).

- Calcificada: placa con señal de baja intensidad, baja atenuación, bordes bien definidos y clara transición entre la capa íntima y el calcio (fig. 1D).

Como es de esperarse hay placas de caracterización compleja y es cuando nacen, a partir de las definiciones básicas, placas mixtas como las fibrolipídicas o fibrocalcificadas.
Varios han sido los intentos por realizar una clasificación de las fases de progresión de la enfermedad coronaria aterosclerótica. El primer consenso de la Asociación Americana del Corazón fue publicado en 1995, e intentó describir seis fases de progresión centradas en el desarrollo del fibroateroma ${ }^{8}$; sin embargo, falló en describir mecanismos importantes dentro de la progresión como las lesiones precursoras, y lesiones trombóticas diferentes a la placa rota que es la lesión culpable de síndrome coronario agudo más común, como la erosión que le sigue en frecuencia en un $30 \%$ de los casos y el nódulo calcificado eruptivo que representa la tercera causa con un $5 \%$.

La clasificación más utilizada al día de hoy fue publicada en 2016 e intenta congregar las diferentes placas en grupos morfológicos ${ }^{9}$ :

- Lesiones no ateroscleróticas de la capa íntima: cambio morfológico aislado de la capa íntima sin alteración de la lámina elástica interna.

- Engrosamiento intimal: lesiones que fisiológicamente son la respuesta al flujo sanguíneo, con engrosamiento adaptativo o difuso de la capa íntima.

- Xantoma intimal: infiltración de la íntima por células espumosas y macrófagos sin disrupción de la lámina elástica interna ni infiltración lipídica. No es considerada una lesión aterosclerótica progresiva.

- Lesiones ateroscleróticas progresivas: pérdida progresiva de la arquitectura trilaminar de la arteria coronaria normal.

- Engrosamiento intimal patológico: lesión más temprana del proceso aterosclerótico conformada por infiltración de la capa íntima por una matriz extracelular con células espumosas, colágeno, proteoglicanos y lípidos. La matriz extracelular puede evidenciar inflamación en mayor o menor grado con infiltración por macrófagos (fig. 2A).

- Fibroateroma: lesión que representa el establecimiento de la aterosclerosis y a partir de la cual nacen diferentes estadios de progresión, caracterizada por un núcleo necrótico generado por infiltración de lípidos y macrófagos. La fase temprana se caracteriza por infiltración de macrófagos (fig. 2D) pero mantenimiento de la matriz extracelular. La fase tardía se determina, por el contrario, por deficiencia de la matriz extracelular y formación de cristales de colesterol (fig. 2E), calcificaciones, hemorragia intraplaca y neovascularización (fig. 2F). Los fibroateromas están encubiertos por una capa fibrosa de colágeno, propensa a adelgazarse y romperse (fig. 2B).

- Fibroateroma con capa fibrosa delgada (reconocida ampliamente por su sigla en inglés TCFA): lesión lipídica vulnerable a la rotura. En general, tiene un núcleo necrótico extenso encubierto por una capa fibrosa muy delgada $<65 \mu \mathrm{m}$ infiltrada por macrófagos, linfocitos y ausencia de células espumosas (fig. 2C).

- Lesiones con trombosis aguda: incluye las lesiones responsables de síndrome coronario agudo.

- Placa rota: es la causa más frecuente de trombosis coronaria, culpable de síndrome coronario agudo en $65 \%$ de los casos. Habitualmente la lesión subyacente es un fibroateroma de capa fibrosa delgada con un núcleo necrótico extenso que se rompe creando una cavidad; al exponerse 

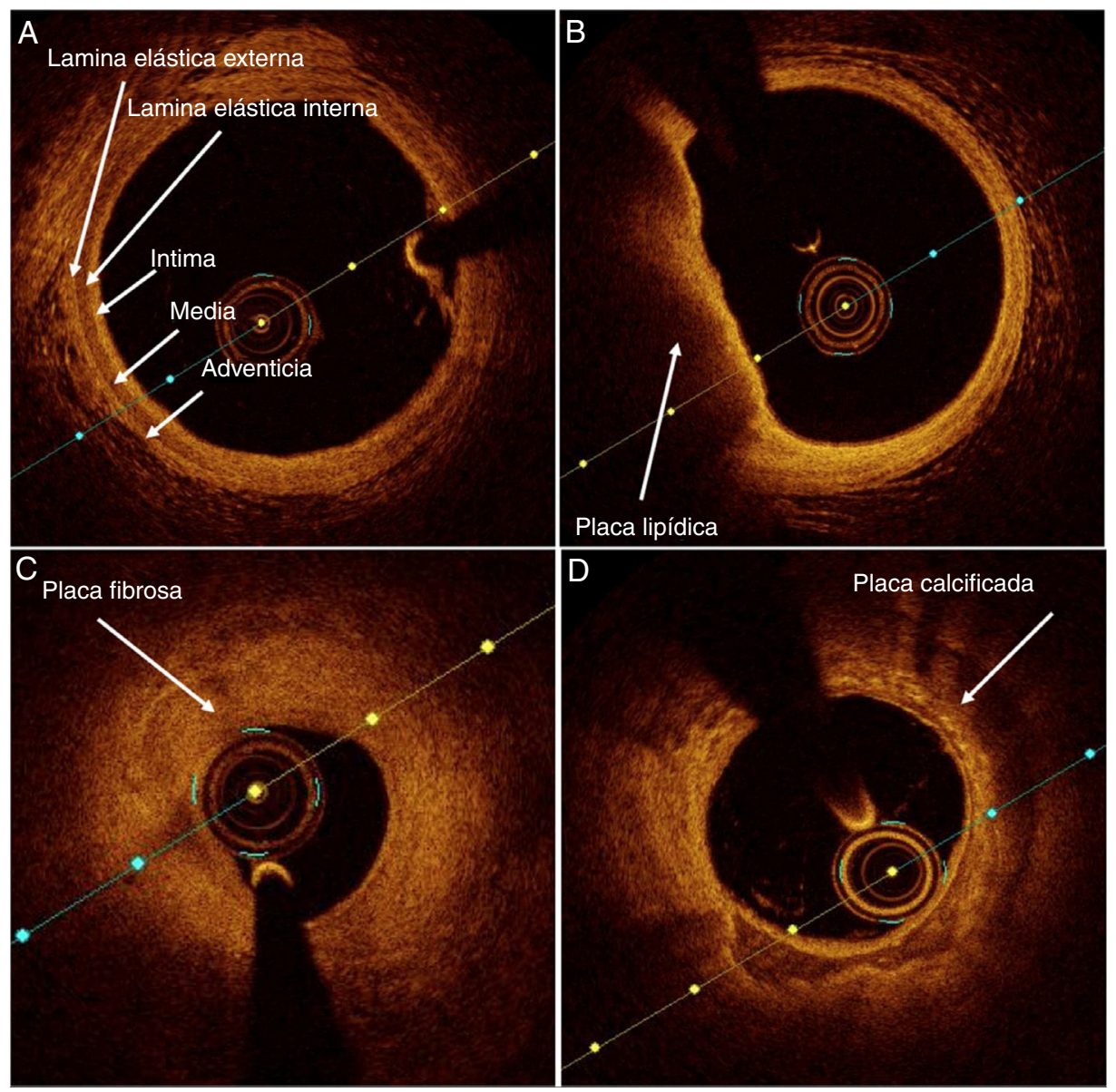

Figura 1 Estructura y conformación de la arteria coronaria normal y las principales placas coronarias. (A) Arteria coronaria normal con arquitectura trilaminar correspondiente a íntima, media y adventicia separadas por láminas elásticas interna y externa. (B) Placa lipídica con señal de alta intensidad, alta atenuación y transición difusa. (C) Placa fibrosa homogénea con señal de alta intensidad y baja atenuación. (D) Placa calcificada con señal de baja intensidad, baja atenuación y bordes bien definidos.

a la luz produce un trombo blanco rico en plaquetas (fig. 3A).

- Placa erosionada: es la segunda causa de trombosis coronaria con una frecuencia del $30 \%$. Se caracteriza por trombo en directo contacto con una superficie intimal denudada con capa fibrosa intacta. La lesión subyacente es generalmente engrosamiento patológico de la íntima o fibroateroma en estados iniciales (fig. 3B).

- Nódulo calcificado eruptivo: es causa de trombosis coronaria en un $5 \%$. Constituye una placa proveniente de una lesión con calcificación severa. La hipótesis propuesta de formación se da a partir de microrroturas debidas a estrés mecánico de la capa que permiten que pequeños nódulos de fibrina erupcionen a la luz y formen un gran nódulo que invade el lumen de la arteria coronaria.

- Lesiones complicadas con hemorragia y/o trombosis tras cicatrización: son lesiones morfológicas resultado de placas complicadas que han cicatrizado y son clínicamente estables.

- Placa rota cicatrizada: rotura de la capa fibrosa que cicatriza y hay ausencia de trombosis coronaria aguda. La mayoría de las placas que se rompen producen trombosis coronaria no oclusiva que no tiene potencial de generar un síndrome clínico. La teoría de la progresión de la aterosclerosis coronaria se basa en el proceso de cicatrización y remodelado negativo que producen estenosis coronaria de forma progresiva. La literatura sugiere que la mayoría de las roturas de placa son asintomáticas ${ }^{10}$ (fig. 4A).

- Trombosis luminal recanalizada: la trombosis coronaria oclusiva eventualmente puede recanalizarse gracias al proceso de infiltración por tejido conectivo y proteoglicanos que producen múltiples canales interconectados, separados por septos de tejido fibroso (fig. 4B).

- Trombosis luminal oclusiva cicatrizada: la trombosis coronaria también puede cicatrizar convirtiéndose en una placa fibrosa oclusiva que da origen a las oclusiones totales crónicas.

- Placa con calcificación severa: la calcificación coronaria está asociada con factores de riesgo como la edad, diabetes, niveles altos de vitamina $D$, alteraciones en el metabolismo óseo y enfermedad renal. La mayoría de las lesiones calcificadas mantienen estables en el tiempo y tienen más bajo potencial de generar un síndrome clínico en comparación con las placas fibroateromatosas (fig. 4C). 

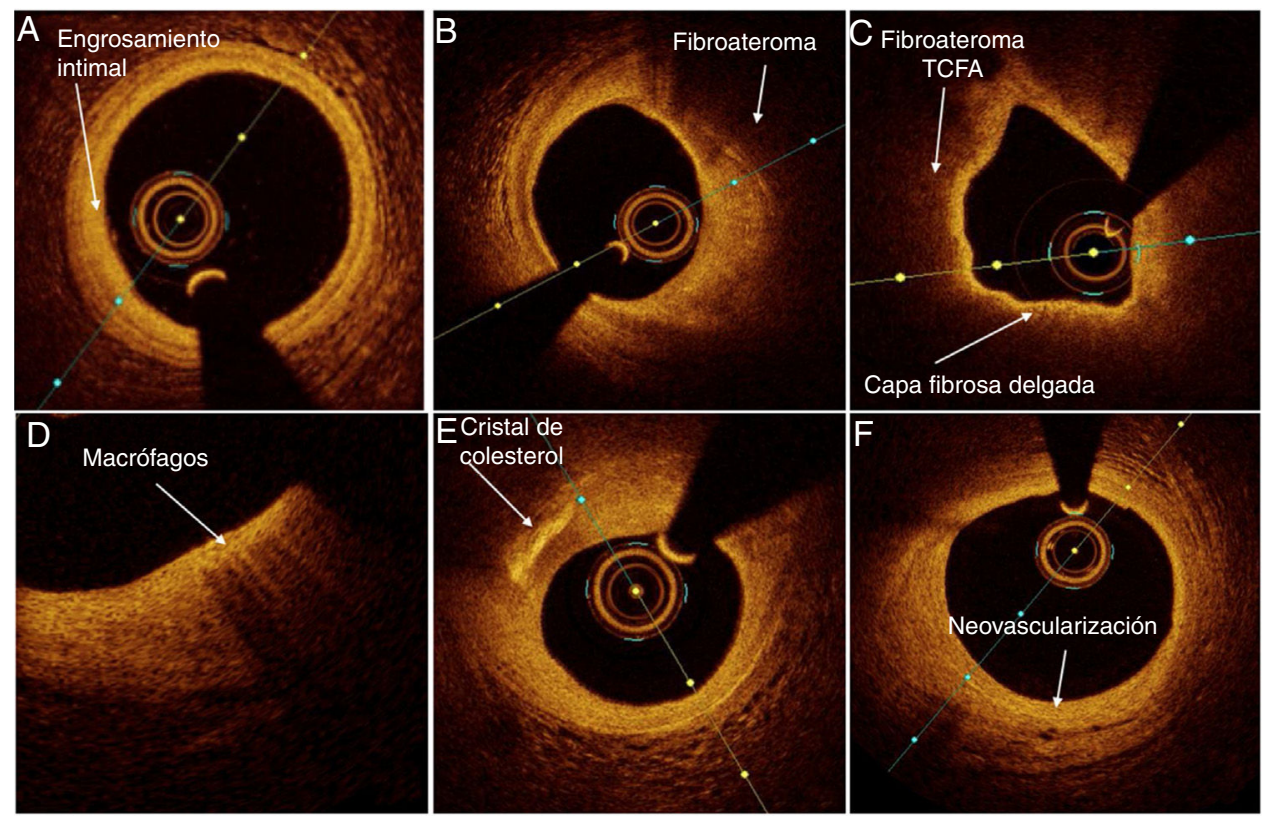

Figura 2 Lesiones ateroscleróticas progresivas. (A) Engrosamiento intimal patológico: lesión temprana del proceso aterosclerótico. (B) Fibroateroma: lesión caracterizada por un núcleo necrótico con infiltración de lípidos y macrófagos. (C) Fibroateroma con capa fibrosa delgada (TCFA): lesión lipídica vulnerable a la rotura con capa fibrosa muy delgada <65 $\mu$ m. (D) Macrófagos. (E) Cristal de colesterol. (F) Neovascularización.
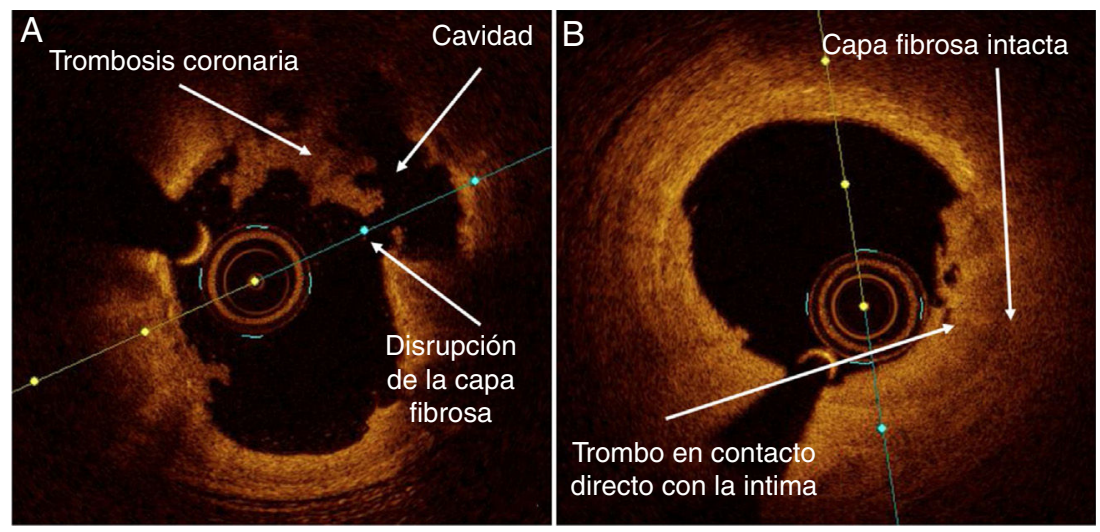

Figura 3 Lesiones con trombosis agudas, responsables del síndrome coronario agudo. (A) Placa rota: fibroateroma de capa fibrosa muy delgada con un núcleo necrótico extenso que se rompe, crea una cavidad y produce trombosis coronaria. (B) Placa erosionada: trombo en contacto directo con la superficie intimal con capa fibrosa intacta.

El reconocimiento morfológico de las lesiones subyacentes por imagen intracoronaria con tomografía de coherencia óptica, se ha convertido en una herramienta muy útil en la práctica clínica habitual, reconociendo trombosis coronaria inaparente a la angiografía y permitiendo la estratificación de las lesiones en estables o inestables. Podemos recrear diferentes situaciones clínicas en las que la tomografía de coherencia óptica puede cambiar la actitud terapéutica:

- Presentación clínica sugerente de miocardiopatía de estrés con lesiones angiográficas que podrían también explicar las alteraciones de la contractibilidad.

- Pacientes sin factores de riesgo cardiovascular y bajo potencial de enfermedad coronaria aterosclerótica que tienen lesiones coronarias sin disección coronaria aparente, en las cuales el diagnóstico de disección coronaria espontánea requiere ser esclarecido para prevenir un intervencionismo coronario innecesario con potencial resultado subóptimo y que puede empeorar el pronóstico. Cuando la angiografía demuestra claramente la disección coronaria o caracteristicas de alta sospecha; el intervencionismo y la imagen intracoronaria no estan recomendados, ya que podrian propiciar la diseminacion de la disección y el empeoramiento del hematoma intramural (fig. 5).

- Pacientes con antecedentes de enfermedades inflamatorias autoinmunes en las que es necesario el diagnóstico diferencial de enfermedad aterosclerótica vs. vasculitis, ya que cambian de forma radical la conducta terapéutica y el pronóstico. 

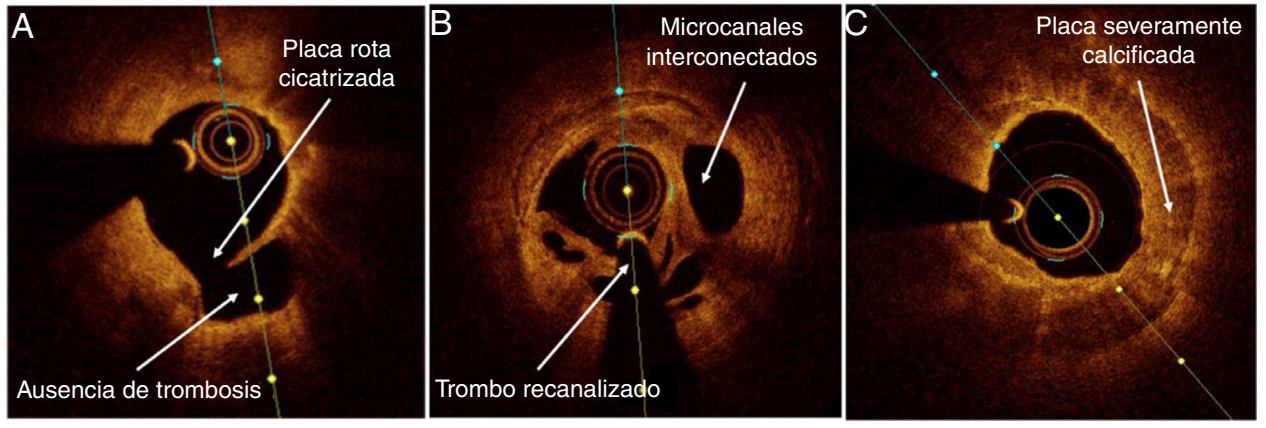

Figura 4 Lesiones complicadas con hemorragia y/o trombosis tras cicatrización. (A) Placa rota cicatrizada: capa fibrosa rota en ausencia de trombosis coronaria. (B) Trombosis luminal recanalizada: múltiples canales interconectados, separados por septos de tejido fibroso. (C) Placa con calcificación severa concéntrica.

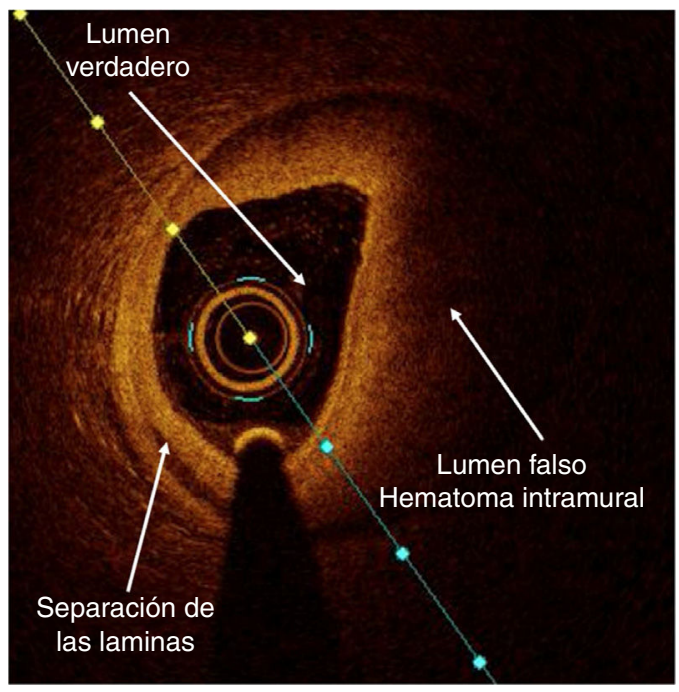

Figura 5 Disección coronaria y hematoma intramural.

En la actualidad, la angiografía orienta la mayoría del diagnóstico e intervencionismo coronario; sin embargo, hay situaciones clínicas agudas en las que es necesario realizar imagen intracoronaria para llegar a una aproximación causal. Claramente, la imagen intracoronaria, y en especial, la tomografía de coherencia óptica por su alta resolución y la capacidad de reconocer trombosis coronaria y placas vulnerables, ha ganado su lugar en el ámbito del síndrome coronario agudo y tiene repercusión diagnóstica y terapéutica.

\section{Fantasía y realidad del proceso de aterosclerosis coronaria}

La aterosclerosis coronaria es un proceso bastante complejo con un espectro morfológico y clínico muy amplio, que va desde lesiones precursoras con solo engrosamiento intimal que al erosionarse tienen el potencial de causar un síndrome coronario agudo, hasta lesiones con múltiples placas rotas cicatrizadas o lesiones con remodelado negativo y oclusiones crónicas que permanecen estables y no presentan ningún síndrome clínico.

La imagen intracoronaria ha desempeñado un papel fundamental en la descripción morfológica y en el entendimiento de la aterosclerosis como una enfermedad difusa que ha sido infraestimada por la angiografía por décadas. Pese a que la imagen intracoronaria ponga de manifiesto la detección de placas vulnerables propensas a la rotura, enfocar la conducta terapéutica en ellas, sería estrechar negligentemente la atención terapéutica y obviar la complejidad y extensión del riesgo potencial; el pronóstico del paciente está dado por la carga de enfermedad aterosclerótica y no por el control de placas ateroscleróticas individuales.

El mecanismo que conduce a un síndrome coronario agudo es mucho más complejo de lo que se asumió inicialmente y depende de la susceptibilidad y adaptación individual al proceso inflamatorio y trombogénico derivado de una placa rota o erosionada con trombosis coronaria. De las muchas placas que se rompen, pocas tendrán el potencial de generar un síndrome clínico, lo que hace difícil la predicción de eventos futuros basados en el análisis de lesiones individuales.

\section{Discusión}

Sin duda el intervencionismo de lesiones inestables y trombóticas oclusivas condicionan un mejor pronóstico para nuestros pacientes; sin embargo, es incierta la necesidad de intervenir lesiones angiográficamente significativas pero clínicamente estables, ya que los eventos futuros pueden recrearse también en lesiones no severas angiográficamente pero con placas vulnerables subyacentes propensas a la rotura $^{11-15}$.

Las nuevas guías europeas de revascularización miocárdica de 2018 recomiendan la revascularización rutinaria de lesiones no relacionadas con la lesión culpable de infarto en el contexto de un síndrome coronario agudo con elevación del segmento ST antes del alta hospitalaria, con clase lla y nivel de evidencia $A^{16-20}$. No obstante, con fundamento en el conocimiento del concepto de placa vulnerable que ha puesto de manifiesto la tomografía de coherencia óptica, es controvertido afirmar que interviniendo las lesiones que son significativas angiográficamente se está protegiendo a los pacientes de eventos futuros, cuando la realidad es que puede haber lesiones vulnerables potenciales causantes de síndromes clínicos en sitios remotos sin severidad angiográfica. El COMPLETE es un estudio en marcha con el objetivo de determinar si realmente la estrategia de 
revascularización multivaso de lesiones no culpables de infarto es superior al tratamiento médico óptimo; incluye también un subestudio con imagen intracoronaria de tomografía de coherencia óptica de las lesiones no culpables, que proporcionará evidencia importante de la morfología subyacente de estas lesiones.

Por décadas se han clasificado los síndromes clínicos como estables e inestables, pero los estudios fisiopatológicos y la imagen intracoronaria han puesto de manifiesto que no hay proceso benigno subyacente en la enfermedad coronaria "estable" o clínicamente silente, ya que la estenosis coronaria proviene del remodelado negativo recurrente de múltiples roturas subclínicas cicatrizadas. Esta consideración abre un espacio para reflexionar sobre el enfoque clínico y terapéutico de ambas situaciones como entidades clínicas distantes.

\section{Conclusiones}

La imagen intracoronaria con tomografía de coherencia óptica ha permitido la apreciación in vivo del proceso de la aterosclerosis y la clasificación de las placas coronarias. Hoy en día es una técnica útil en la práctica clínica habitual para diagnóstico de la lesión culpable en casos en los que la angiografía es limitada, en la planificación de intervencionismo percutáneo complejo y ante falla del stent.

\section{Bibliografía}

1. Topol E, Nissen S. Our preoccupation with coronary luminology. The dissociation between clinical and angiographic findings in ischemic heart disease. Circulation. 1995;92:2333-42.

2. Zir LM, Miller SW, Dinsmore RE, Gilbert JP, Harthorne JW. Interobserver variability in coronary angiography. Circulation. 1976;53:627-32.

3. Yong AS, Ng AC, Brieger D, Lowe HC, Ng MK, Kritharides L. Three-dimensional and two-dimensional quantitative coronary angiography and their prediction of reduced fractional flow reserve. Eur Heart J. 2011;32:345-53.

4. Glagov S, Weisenberg E, Zarins CK, Stankunavicius R, Kolettis GJ. Compensatory enlagerment of human atherosclerotic coronary arteries. N Engl J Med. 1987;316:1371-5.

5. Mintz GS. Clinical utility of intravascular imaging and physiology in coronary artery disease. J Am Coll Cardiol. 2014;64:207-22.

6. Prati F, Guagliumi G, Mintz GS, Costa M, Regar E, Akasaka T, et al. Expert review document on methodology, terminology, and clinical applications of optical coherence tomography: physical principles, methodology of image acquisition, and clinical application for assessment of coronary arteries and atherosclerosis. Eur Heart J. 2010;31:401-15.

7. Prati F, Regar E, Mintz GS, Arbustini E, Di Mario C, Jang IK, et al. Expert review document part 2: methodology, terminology and clinical applications of optical coherence tomography for the assessment of interventional procedures. Eur Heart J. 2012;33:2513-20.

8. Stary HC, Chandler AB, Dinsmore RE, Fuster V, Glagov S, Insull $W$, et al. A definition of advanced types of atherosclerotic lesions and a histological classification of atherosclerosis. A report from the Committee on Vascular Lesions of the Council on Arteriosclerosis, American Heart Association. Circulation. 1995;92:1355-74.

9. Yahagi K, Kolodgie FD, Otsuka F, Finn AV, Davis HR, Joner M, et al. Pathophysiology of native coronary, vein graft, and instent atherosclerosis. Nat Rev Cardiol. 2016;13:79-98.

10. Mann J, Davies MJ. Mechanisms of progression in native coronary artery disease: role of healed plaque disruption. Heart. 1999;82:265-8.

11. Little WC, Constantinescu M, Applegate RJ, Kutcher MA, Burrows MT, Kahl FR, et al. Can coronary angiography predict the site of a subsequent myocardial infarction in patients with mild-to-moderate coronary artery disease? Circulation. 1988;78:1157-66.

12. Ambrose JA, Tannenbaum MA, Alexopoulos D, Hjemdahl-Monsen CE, Leavy J, Weiss M, et al. Angiographic progression of coronary artery disease and the development of myocardial infarction. J Am Coll Cardiol. 1988;12:56-62.

13. Glaser R, Selzer F, Faxon DP, Laskey WK, Cohen HA, Slater J, et al. Clinical progression of incidental, asymptomatic lesions discovered during culprit vessel coronary intervention. Circulation. 2005;111:143-9.

14. Stone GW, Maehara A, Lansky AJ, de Bruyne B, Cristea E, Mintz GS, et al. A prospective natural-history study of coronary atherosclerosis. N Engl J Med. 2011;364:226-35.

15. Niccoli G, Stefanini GG, Capodanno D, Crea F, Ambrose JA, Berg R. Are the culprit lesions severely stenotic? JACC CardiovasC Imaging. 2013;6:1108-14.

16. Wald DS, Morris JK, Wald NJ, Chase AJ, Edwards RJ, Hughes LO, et al. Randomized trial of preventive angioplasty in myocardial infarction. PRAMI Investigators. N Engl J Med. 2013;369:1115-23.

17. Gershlick AH, Khan JN, Kelly DJ, Greenwood JP, Sasikaran T, Curzen N, et al. Randomized trial of complete versus lesion-only revascularization in patients undergoing primary percutaneous coronary intervention for STEMI and multivessel disease: The CVLPRIT trial. J Am Coll Cardiol. 2015;65:963-72.

18. Engstrom T, Kelbaek H, Helqvist S, Hofsten DE, Klovgaard L, Holmvang $L$, et al. Complete revascularisation versus treatment of the culprit lesion only in patients with ST-segment elevation myocardial infarction and multivessel disease (DANAMI-3-PRIMULTI): An open-label, randomised controlled trial. Lancet. 2015;386:665-71.

19. Smits PC, Abdel-Wahab M, Neumann FJ, Boxma-de Klerk BM, Lunde K, Schotborgh CE, et al. Fractional flow reserve-guided multivessel angioplasty in myocardial infarction. CompareAcute Investigators. N Engl J Med. 2017;376:1234-44.

20. Sousa-Uva M, Neumann FJ, Ahlsson A, Alfonso F, Banning AP, Benedetto $U$, et al. ESC/EACTS Guidelines on myocardial revascularization. Eur J Cardiothorac Surg. 2018;55:4-90. 